Cite as: Fujita, T. and Jones, K. (2007), Learners' understanding of the definitions and hierarchical classification of quadrilaterals: towards a theoretical framing, Research in Mathematics Education, 9(1\&2), 3-20. ISSN: 1479-4802; ISBN: 0953849880

\title{
LEARNERS' UNDERSTANDING OF THE DEFINITIONS AND HIERARCHICAL CLASSIFICATION OF QUADRILATERALS: TOWARDS A THEORETICAL
} FRAMING

\author{
Taro Fujita, University of Plymouth, UK
}

Keith Jones, University of Southampton, UK

Defining and classifying quadrilaterals, though an established component of the school mathematics curriculum, appears to be a difficult topic for many learners. The reasons for such difficulties relate to the complexities in learning to analyse the attributes of different quadrilaterals and to distinguish between critical and noncritical aspects. Such learning, if it is to be effective, requires logical deduction, together with suitable interactions between concepts and images. This paper reports on an analysis of data from a total of 263 learners. The main purpose of the paper is to present a theoretical framing that is intended to inform further studies of this important topic within mathematics education research. This theoretical framing relates prototype phenomenon and implicit models to common cognitive paths in the understanding of the relationship between quadrilaterals.

\section{INTRODUCTION}

The teaching of geometry provides not only a key vehicle for developing learners' spatial thinking and visualisation skills, but also a major opportunity to develop their ability in deductive reasoning and proving (Battista, 2007; Royal Society, 2001). In this latter aspect of geometry teaching, that of developing deductive reasoning and proving, the notion of definitions is very important, especially given the role of definitions in identifying, with some precision, new mathematical objects. In this way, definitions assign properties to mathematical objects.

Intimately related to the notion of definition, and just as important in mathematics, are ideas of classification (linked to notions of isomorphism). One of the reasons for the importance of classification in the teaching and learning of mathematics is captured in the following words of the renowned mathematician Henri Poincaré:

The definition will not be understood until you have shown not only the object defined, but the neighbouring objects from which it has to be distinguished, until you have made it possible to grasp the difference, and have added explicitly your reason for saying this or that in stating the definition. (Poincaré, 1914, p. 452)

Echoing the importance of such issues, a key focus for mathematics education research concerns learners' understanding both of definitions and of classification, given that a range of studies is indicating that learners have difficulties both with defining (see, for example, de Villiers, 1998; Vinner, 1991; Zaslavsky and Shir, 2005) and classifying (see, for instance, Currie and Pegg, 1998; de Villiers, 1994; Monaghan, 2000). Given the importance of this issue, the purposes of this paper are, 
first, to report findings concerning learners' knowledge of the definitions of, and classification relationships between, quadrilaterals, and, second, to propose a theoretical framing that is intended to inform further analyses in this research area.

\section{THE HIERARCHICAL CLASSIFICATION OF QUADRILATERALS}

In mathematics there is a general preference for a hierarchical classification of quadrilaterals and school curricula usually follow this approach, in particular, at the lower secondary school level (for more on this, see de Villiers, 1994). One reason for the preference for a hierarchical classification is its 'economical' character in that, for example, if a statement is true for parallelograms, this means that it is also true for squares, rectangles and rhombuses as these may be described as 'special' types of parallelograms.

A number of international studies have shown that many learners have problems with a hierarchical classification of quadrilaterals and the related issue of defining such shapes (Currie and Pegg, 1998; de Villiers, 1994; Erez and Yerushalmy, 2006; Monaghan, 2000; Pickreign, 2007). In particular, it is evident that learners often have difficulties with the formal definitions of shapes and, further, that their geometrical reasoning is often significantly influenced by their mental images of shapes. For example, Monaghan (2000) reported that the 'horizontal length' of typical images of rectangles disturbs learners' perceptions of 'inclusion relationships' of quadrilaterals, and that 11 year old students in the UK are not likely to accept that a square is a special type of rectangle (p. 186). Such difficulties, as Erez and Yerushalmy (2006, p. 272) explain (following the work of Markman, 1991), are related to the complexities in learning to analyse the attributes of different quadrilaterals and to distinguish between critical and non-critical attributes of different ones such that understanding hierarchical relations involves at least the following:

- the ability to classify a shape in different ways and label it with different names; for example, that a rhombus can also be called a polygon, a quadrilateral, a special type of parallelogram or kite, and so on;

- the need to understand the transitive relations between the concepts of shapes; for example, that if a square is a rhombus and a rhombus is a parallelogram, then a square is also a parallelogram;

- the need to understand the asymmetry of relations among quadrilaterals; for example, that every rectangle is a parallelogram, but not every parallelogram is a rectangle;

- the need to understand the opposite asymmetry and transitive relations of the critical attributes of shape concepts: for example, that the critical attributes of the rectangle are included in the critical attributes of the square, but the critical attributes of the square are not included in those of the rectangle.

This difficulty with definitions and classification appears to persist with trainee elementary school teachers (perhaps, in some ways, unsurprisingly) even though they 
are expected to have a sound knowledge of this area of mathematics in order to teach the topic effectively. For example, Kawasaki (1992) conducted a survey of Japanese trainee elementary school teachers' knowledge of quadrilaterals (parallelogram, rectangle, square and trapezium) and found that just $5 \%$ of his sample $(\mathrm{N}=56)$ could write a formal definition of a rectangle, and that many of the trainees (in their first year of university study) used their own image of rectangles for their definition, such as 'a rectangle is a quadrilateral whose sides are different lengths'. Similar findings are reported by Pickreign (2007) in the case of trainee teachers in the USA, while there are indications that there might be comparable issues in the UK (see, for instance, Jones, Mooney and Harries, 2002).

In the next section, we discuss further the complexities both of geometrical figures and of reasoning about the relationships among them. In particular, we explore why the hierarchical classification is difficult for many learners in terms of the van Hiele theory, and the relationship between images and definitions of geometrical figures.

\section{THEORETICAL CONSIDERATIONS}

The van Hiele model for the learning of geometry, which suggests that learners advance through levels of thought in geometry (Crowley, 1987; van Hiele, 1999), is generally considered to be a fairly useful model to describe learners' behaviours in geometry (Battista, 2007; Senk, 1989). The model specifies the following levels (we are using the 1-5 numeration, following authors such as Battista, 2007):

- Level 1: Visual - identifying shapes according to their concrete examples

- Level 2: Descriptive/analytic - identifying shapes according to their properties

- Level 3: Abstract/relational/informal deduction - identifying relationships between shapes and producing simple logical deduction

- Level 4: Formal deduction - understanding logical deduction

- Level 5: Rigor/meta-mathematical - axiomatic systems of geometry are understood.

In terms of this model, learners at van Hiele level 3 are, for example, expected to be able to deduce that a rectangle is a special type of parallelogram by considering definitions and properties of these quadrilaterals. Learners at level 2 start recognising properties of individual shapes (for example, that in a square all the sides are the same and that all the angles are the same), while learners at level 1 would recognise a square or rectangle from their overall shape and that they are different from a circle. Research evidence suggests that the rate of progress from level 2 to 3 made by many school children and students is slow, or even that many of them remain at level 2 by the end of the secondary (high) school (Senk, 1989). Thus, the hierarchical classification of quadrilaterals, taken to be van Hiele level 3, can be regarded as a difficult task for many learners.

While the van Hiele model provides us with insights into why the hierarchical classification is difficult, we should also explore the cause of these difficulties in 
terms of the nature of geometrical figures. The terms 'concept image' and 'concept definition' were introduced by Vinner and Hershkowitz (1980) in the context of the learning of some simple geometrical concepts, and developed by Tall and Vinner (1981) in the context of more sophisticated mathematical ideas of limits and continuity. Given that formal concept definitions are definitions that are accepted as mathematical, Tall and Vinner (1981, p. 152) defined a concept definition as 'a form of words used to specify that concept' and concept image as 'the total cognitive structure that is associated with the concept, which includes all the mental pictures and associated properties and process'. In terms of geometrical figures a characteristic feature is their dual nature, in that both concept and image are closely inter-related. In this context, Fischbein (1993) proposed the notion of 'figural concept' in that, while a geometrical figure (such as a square) can be described as having intrinsic conceptual properties (in that it is controlled by geometrical theory), it is not solely a concept; it is also an image (p. 141). Thus, when considering a square, it can be regarded as 'a quadrilateral whose sides and angles are equal (a concept)' as well as $<\square>$ (an image) and not $<\square>$.

Taking this approach, and from a purely cognitive perspective, on the one hand individual learners can be thought of as having their own concept images and their personal concept definitions of basic figures, all constructed through their own experiences of learning geometry; for the purposes of analysis in this paper, we call examples of these personal figural concepts. On the other hand, there are formal concept images and definitions in geometry such that, when Euclidean definitions are used, a square, for instance, is defined as a quadrilateral whose sides and angles are equal; we call such an example a formal figural concept.

When we classify quadrilaterals, we exercise our own personal figural concepts, and the result depends on what personal figural concepts of quadrilaterals we have. This means, for example, that if one's personal figural concepts are not fully developed, as Vinner (1991) suggests can happen, one's judgement would likely be influenced by 'images' (perhaps in the van Hiele level 1 sense) only, and this may well result in an incorrect (from a mathematical point of view) conclusion. For example, if one's personal figural concept of parallelograms excludes rhombuses from the images of parallelograms, one may not accept a rhombus is a special type of parallelogram.

These theoretical considerations suggest that the hierarchical classification is difficult for many learners because of the complex nature of figural concepts of geometrical shapes. These complexities also make it difficult for mathematics educators (teachers, teacher trainers, curriculum researchers, researchers and so on) to provide useful suggestions to improve the situation in the learning and teaching of geometry. Our goal is to contribute to these matters.

\section{RESEARCH DESIGN}

As a starting point for research into learners' understanding of the classification of quadrilaterals, it is valuable to find out at what level of thinking they are in terms of 
van Hiele's model, and what personal figural concepts they have of quadrilaterals. Having established this, a further aim is to explore the nature of any 'gap' between personal figural concepts and formal figural concepts (that is, the 'gap' between the actual knowledge learners appear to have, and the knowledge that educators expect learners to have).

This study is a part of a larger study being carried out in collaboration between researchers in the UK and in Japan, and the data presented in this paper is from an opportunistic sample of trainee elementary school teachers on a four-year teacher training course in Scotland. We consider that it is valid to examine the knowledge of trainee teachers as this group are also 'learners' (in addition to being future classroom educators) in that they are still developing their knowledge to teach mathematics in schools, and, as discussed in the previous section, seem likely to display similar forms of understanding (and difficulties) as school-age learners. Further details of the research design, together with the results from our analysis of two suitable sets of data, are presented in the next section.

\section{LEARNERS' KNOWLEDGE OF QUADRILATERALS}

\section{Personal figural concepts of quadrilaterals}

The first set of data we consider comes from a survey of 158 learners (noting, as mentioned above, that, in this particular component of our study, they are trainee elementary school teachers in their first year of university study, and that most were 18 years old at the time of data collection). These learners were asked, following some taught input designed to remind them of the basic properties of and the relationships between quadrilaterals, to complete the questionnaire set out in Table 1 .

The purpose of this questionnaire was to reveal any 'gap' between the formal and personal figural concepts of the learners. The design of this element of the study was informed by the research of Kawasaki (1992) mentioned above, which investigated possible gaps in trainee teachers' figural concepts of quadrilaterals in Japan by using the questions in Table 1.

Analysis of data from responses to question 1 indicates that there are, indeed, 'gaps' in the learners' knowledge in this area. For example, 14 out of $158(8.9 \%)$ answered correctly Q1a about whether a square is a trapezium, $20(12.7 \%)$ answered that a square is a rectangle, and $29(18.4 \%)$ answered that a parallelogram is a trapezium (note that, in Kawasaki's study, a trapezium is regarded as a quadrilateral which has at least one pair of parallel lines, and therefore a parallelogram is regarded as a special type of a trapezium; while we follow this definition in our research, we are also aware that alternative definitions may appear in current mathematics textbooks, see Usiskin and Dougherty, 2007). The latter result contrasts sharply with Kawasaki's findings that $73 \%$ of Japanese trainee teachers $(\mathrm{N}=56)$ could define a trapezium correctly. There is a possibility that Scottish trainee teachers in our study might not be familiar with the type of question, while Japanese trainees are familiar with those questions as they have studied the Euclidean style of geometry in lower secondary 
schools (see Fujita and Jones, 2003b).

Table 1: Research questionnaire - Quadrilaterals

Q1. Answer the following questions, and state your reasons briefly.
a. Is a square a trapezium?
b. Is a square a rectangle?
c. Is a parallelogram a trapezium?
Q2. A kite is defined as 'a quadrilateral, which has both pairs of adjacent sides
equal'. Define the following quadrilaterals, and draw an image of each.
a. A parallelogram
b. A square
c. A rectangle
d. A trapezium

The results from Q2 (Table 1) are given in Table 2 and Figure 1 - the former showing the results from, and the latter comparing the numbers of trainees providing the correct image compared to the number providing a correct definition.

Table 2 and Figure 1: Correct responses to Question 2

\begin{tabular}{|l|l|l|}
\hline Q2a Image Parallelogram & $153(96.8 \%)$ & \\
\hline Q2a Definition Parallelogram & $93(58.9 \%)$ \\
\hline Q2b Image of a square & $154(97.5 \%)$ & \\
\hline Q2b Definition of a square & $60(38 \%)$ & 180 \\
\hline Q2c Image of a rectangle & $155(98.1 \%)$ & $34(21.5 \%)$ \\
\hline Q2c Definition of a rectangle & $96(60.8 \%)$ & \\
\hline Q2d Image of a trapezium & $19(12 \%)$ & \\
\hline Q2d Definition of a trapezium & & \\
\hline
\end{tabular}

Comparing the image and definition results displayed in Figure 1 reveals that the majority of the learners could at least draw a correct image of the quadrilaterals (with the exception of a trapezium) but far less were able to provide their definitions. In the theoretical discussion in this paper (see above), it was proposed that images in learners' personal figural concepts have a strong influence when they attempt to define/classify figures, and this appears to be borne out in this study. For example, almost all learners could draw a correct image of a square, while 62\% (98 learners) defined it incorrectly. Of these, 80 (about $82 \%$ of 98) wrote 'a quadrilateral whose sides are equal' and did not refer to 'angles'. If they had fully considered their figural concepts, they should have noticed that a rhombus can also satisfy this condition, and therefore it would be necessary to include something about the angles as well. It could be that the image $<\square>$ is so strong for them that many do not recognise the need to mention the angles being equal.

Similarly, while 155 (98\%) could draw an image of a rectangle, only 34 (21.5\%) could define it correctly. Almost 70\% (86 out of 124) defined a rectangle as 'a quadrilateral which has two longer sides and two shorter sides' (similar results are 
reported, for example, by Monaghan, 2000, p. 186). Again, learners appear to be influenced by the image of a rectangle and omit to mention anything about its angles. Moreover, 68 (43\% of 158) defined both a square and a rectangle without mentioning angles. The results for parallelogram are slightly better, with almost $97 \%$ being able to draw an image of a parallelogram, and almost $60 \%$ being able to define it. While this more positive result could, perhaps, result from the name 'parallelogram' reminding the learners of 'parallel lines', as revealed in the next section of analysis, such an interpretation needs to be treated with some caution as the learners' perceptions/ understanding of parallelograms might be more limited than the data in Figure 1 suggests (for example, the learners might consider a parallelogram to be a 'slanted' quadrilateral and, moreover, that a rectangle is not a parallelogram).

\section{What do learners know about parallelograms?}

Our second set of data comes from a survey of 105 learners (noting that, in this particular study, they are trainee elementary school teachers in their second year of university study, and most were 19 20 years old). The questionnaire we used, see Table 3, was adapted from one originally designed by Koseki (1987, see also Kunimune, 2000) to measure the level of understanding of parallelograms held by equivalent trainees and school children in Japan and it consists of six questions.

Table 3 and Figure 2: Research questionnaire - Parallelogram

Q1. Choose which are parallelograms from the quadrilaterals 1 15 below.

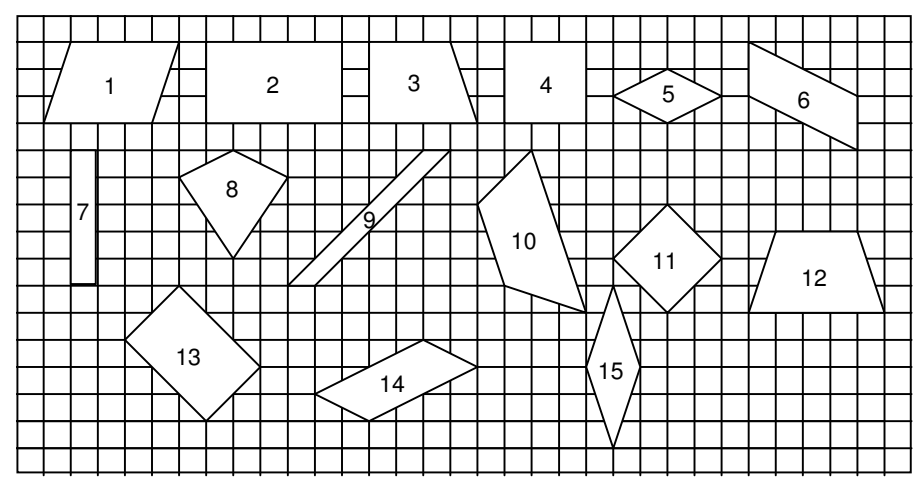

Figure 2: Quadrilaterals for Q1

Q2. What is a parallelogram? Draw its image and describe it in words.

Q3. Is it possible to draw a parallelogram whose four vertices are on the circumference of a circle? Choose your answer a. or b. If you choose (a), state your opinion why it is not possible. If your answer is (b), draw its shape and name in the circle. (a) No it is not possible, because ... or (b) Yes, it is possible.

Q6. Answer whether the following statements are true or false

a. There is no relationship between a rhombus and a parallelogram. True/False

b. It is possible to say that a rhombus is a parallelogram. True/False

c. It is possible to say that a parallelogram is a rhombus. True/False

The studies by Koseki and by Kunimune, independent of studies based on the van Hiele model, are premised on there being several stages in learners' thinking about 
parallelograms; stage 1 for learners who have very limited figural concepts of parallelograms, stage 2 for those who began to extend their figural concepts, (for example that rhombuses are also parallelograms) but still do not have sound understanding, and stage 3 for those who have formal figural concepts of parallelogram with the understanding of class inclusion. Due to limitations of space in this paper, we report on part of our results - those relating to items 1,2, 3 and 6 of the questionnaire.

While Q1 and Q2 check learners' basic knowledge of parallelograms, Q3 asks them to determine parallelograms which can be inscribed in a circle (the answer is rectangles). Thus this question checks whether learners are able to use a hierarchical relationship to solve a problem; that is, whether they can understand that it is not only a square, but also any kind of rectangle, of which a square is just one, which can be inscribed in a circle.

Table 4 summarises the marking criteria for each question from the questionnaire in Table 3. Each question is measured by $0 \sim 3$ points which correspond to the stages described above.

Table 4: Marking criteria for Q1, Q2 \& Q3

\begin{tabular}{|c|l|l|l|l|}
\hline & \multicolumn{1}{|c|}{3 pt. } & \multicolumn{1}{c|}{2 pt. } & \multicolumn{1}{c|}{1 pt. } & 0 pt. \\
\hline Q1 & $\begin{array}{l}\text { At least ten of the } \\
\text { following: } 1,2,4,5, \\
6,7,9,11,13,14,15\end{array}$ & $\begin{array}{l}\text { At least six of 1, 5, 6, } \\
9,11,14,15 \text { or eight } \\
\text { of } 1,2,4,6,7,9,11, \\
13,14,15\end{array}$ & $\begin{array}{l}\text { At least three of the } \\
\text { following: 1, 6, 9, } 14\end{array}$ & Others \\
\hline Q2 & $\begin{array}{l}\text { Correct definition \& } \\
\text { image }\end{array}$ & $\begin{array}{l}\text { Stating too many } \\
\text { properties }\end{array}$ & $\begin{array}{l}\text { Statements involving } \\
\text { limited images (such } \\
\text { as 'slant rectangle') }\end{array}$ & Others \\
\hline Q3 & $\begin{array}{l}\text { (b) and draws \& } \\
\text { names a rectangle }\end{array}$ & $\begin{array}{l}\text { (b) and draws \& } \\
\text { names a rectangle and } \\
\text { a square }\end{array}$ & $\begin{array}{l}\text { (b) and draws a } \\
\text { correct image } \\
\text { (b) and draws \& } \\
\text { names a rhombus }\end{array}$ & Others \\
\hline Q6 & True for b. & $\begin{array}{l}\text { True for a. \& b. (if 2 } \\
\text { or 3 pt in Q1) }\end{array}$ & $\begin{array}{l}\text { True for c. (if 2 or 3 } \\
\text { pt in Q1) }\end{array}$ & Others \\
\hline
\end{tabular}

In Q1, if a student can identify all parallelograms correctly (i.e. 1, 2, 3, 4, 5, 6, 7, 9, $11,13,14$ and 15 in fig. 1), s/he receives ' 3 ' points for Q1 (and is considered, in the Kunimune model, to be at stage 3), whereas a student who chooses very limited images of parallelograms such as 1, 6, 9, 14 receives ' 1 ' point. Some students might be starting to extend their knowledge in parallelograms, and choose, for example, 1, 6, 9, 14, 5, 11 and 15 (or similarly ' $1,2,4,6,7,9,11,13$ and 14', ' $1,2,4,6,9,13$ and $15^{\prime}$ ' and so on) as parallelograms, and those students receive '2' points. In Q2, a student receives ' 3 ' points if s/he states a correct (formal) definition of a parallelogram, whereas a statement such as 'a parallelogram is a slanted quadrilateral' is awarded ' 1 ' point. In Q3, if a student says 'Yes, it is a rectangle' then s/he has 
utilised their knowledge 'a rectangle is a parallelogram' to solve this question, and receives ' 3 ' points. A student who answers either 'Yes, it is a rectangle and a square', or 'Yes, it is a square', is taken as not understanding the inclusion relation between rectangle/square and hence s/he receives ' 2 ' points (either because, in the first case, the square is mentioned unnecessarily, or because, in the second case, the rectangle is omitted altogether - in both these case, the student is taken as not being able to see that 'a square is a (special type of) rectangle').

Table 5 summarises the results of Q1, Q2, Q3 \& Q6

Table 5: Results of Q1, Q2, Q3 \& Q6

\begin{tabular}{|c|c|c|c|c|}
\hline & Q1 & Q2 & Q3 & Q6 \\
\hline $3 \mathrm{pt}$ & $21(20 \%)$ & $59(56.2 \%)$ & $8(7.6 \%)$ & $38(36.2 \%)$ \\
\hline $2 \mathrm{pt}$ & $27(25.7 \%)$ & $27(25.7 \%)$ & $11(10.5 \%)$ & $0(0 \%)$ \\
\hline $1 \mathrm{pt}$ & $47(44.8 \%)$ & $11(10.5 \%)$ & $10(9.5 \%)$ & $4(3.8 \%)$ \\
\hline $0 \mathrm{pt}$ & $10(9.5 \%)$ & $8(7.6 \%)$ & $76(72.4 \%)$ & $63(60 \%)$ \\
\hline
\end{tabular}

The results for Q2 show that over 80\% of the 105 learners in the second data set could define a parallelogram almost correctly, and generally they could draw a correct image of it. However, as the result of Q1 indicates, it is likely that their personal figural concepts of parallelograms consist of a correct concept definition and limited images (such as 'leaned' parallelograms, Monaghan, 2000, p. 188), given that just $20 \%$ could identify all correct images of parallelograms - 47 (44.8\%) chose images 1, 6, 9 and 14 (in Figure 2). This implies that almost half of the learners still regard parallelograms in terms of limited images despite the fact that they have a fair understanding of the concept definition. Thus, there is a 'gap' between their formal and personal figural concepts of parallelograms.

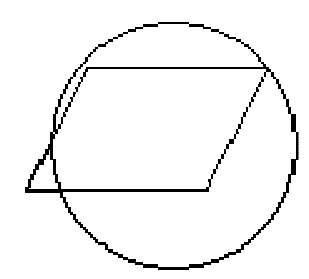

Figure 3: One form of answer to Q3

The performance in terms of Q3 shows the learners' limitations with this topic. While just 8 of $105(7.6 \%)$ could answer that 'it is a rectangle which satisfies the statement in Q3', 76 (72.4\%) answered 'No, it is not possible'. Of these 76, 15 reasoned their answer by stating 'you will always get a right angle if you draw a parallelogram in a circle' or 'there will be only 2 vertices which touch', 5 merely drew an image (see Figure 3), 20 stated their reasons and drew an image, and 20 gave no answer. In other words, 40 of 76 learners who scored '0' points in Q3 used 'slanted' images of a parallelogram to tackle this question (we discuss this issue later in this paper in terms 
of 'prototype phenomenon').

We determined a slight correlation between the performances in relation to these questions. The value of Pearson's correlation coefficient between Q1 and Q3 is $\mathrm{r}=0.48 \quad(\mathrm{p}<0.01)$; that is, learners who could choose the correct images of parallelograms were likely to get better scores than those who just chose the limited images of parallelograms. A reason for why there is not a stronger correlation is that 6 learners out of the 21 who scored '3' points, and 18 of 28 who scored '2' points for Q1, could not answer Q3 correctly, perhaps because their ability to control their images still does not appear consolidated when they try to solve geometrical problems.

In Q1, 27 learners scored '2' points, and 17 of them (about 63\% of 27) included at least one of the images of rectangles (e.g. 2, 7, 13 in Figure 2) or squares (4 or 11 in Figure 2) as parallelograms. The others chose the 'typical' images of parallelograms and rhombuses $(1,6,9,14+5,15$ in Figure 2). Interestingly, 8 of these 17 could achieve ' 1 ', '2', or even ' 3 ' points for Q3. This implies that one of the important factors in solving Q3 is what images of parallelograms they are able to utilise (or how they can exercise their 'geometrical eye', see Fujita and Jones, 2003a).

Q6 checks whether the learners could see a rhombus as a parallelogram. The results point to a lack of understanding about the relationship between parallelograms, even for those who could score 2 or 3 points in Q1: the value of Pearson's correlation coefficient between Q1 and Q6 is $\mathrm{r}=0.2(\mathrm{p}<0.01)$. Eight learners who scored 3 points for Q1 chose 'true' for ' $b$ ' and 'c' in Q6. This indicates that, although they could choose correct images of 'parallelograms', they simultaneously considered both ' $b$ ' (a rhombus is a parallelogram) and ' $c$ ' (a parallelogram is a rhombus) to be true. As we speculated may be the case for Q1 in the first data set (table 1), there is a possibility that the learners in our study might not be familiar with the type of question. Considering the findings that our subjects show particular weaknesses in this type of question, in follow-up studies more attention could be paid to how questions are worded. For example, a formulation such as the following could be considered: 'It is possible to say that a rhombus is a special type of parallelogram?'.

\section{TOWARDS A FUTURE THEORETICAL FRAMEWORK}

As has been established, the hierarchical classification of quadrilaterals is difficult because it requires logical deduction, together with suitable interactions between concepts and images. The analysis we present in this paper aligns with related research, confirming the difficulties learners have with coming to an understanding of the hierarchical relationship between quadrilaterals (and, as such, that many remain at level 2 - or even level 1 - in terms of the van Hiele model), and that a 'gap' seems to exist between learners' formal figural concepts and their personal figural concepts such that images are so influential in learners' personal figural concepts that they dominate their attempt to define basic quadrilaterals.

These findings confirm that there are crucial issues to be addressed if there are to be 
improvements in the situation regarding learners' knowledge of geometrical figures. In what follows, both by reflecting on our findings outlined above and through theoretical discussion, we propose a theoretical framing intended to inform future research in this area. To do so we first revisit the van Hiele model and clarify how we might situate the study of the relationships between quadrilaterals within the geometry curriculum. We then focus on related theories in this area, in particular 'tacit models' (Fischbein et al, 1985), 'prototype phenomenon' (Hershkowitz, 1990), and 'common cognitive paths' in geometrical thinking (Vinner and Hershkowitz, 1980).

\section{The study of the hierarchical classification of quadrilaterals as bridging a gap between van Hiele levels 2 and 3}

Hershkowitz (1990) states 'van Hiele's theory takes the goal of geometry learning to be the realization of geometry as a deductive structure, with geometry as the science of our environment as a necessary prerequisite' (p. 73). An important research issue is to investigate how we might design learning and teaching which would promote learners' advancement from one level to another in the van Hiele model. Given that van Hiele calls the stages from levels 1 to 2 and levels 2 to 3 as 'Period 1' and 'Period 2' respectively, and considering existing research findings such as Senk's (1989) study, we focus on 'Period 2' as this is where the transition between levels 2 and 3 occurs.

The van Hiele model suggests that learners at level 2 start recognising the properties of individual shapes, and those at level 3 start simple deduction concerning these properties. Thus the key factor influencing student progress during period 2 is how learners move from regarding 'shapes' as the object of their study through a focus on 'shape properties'/ to regarding 'shape/geometrical properties' as the object of their study through a focus on 'deductive reasoning' (Okazaki and Iwasaki, 2003). This is summarised in Table 6.

Table 6: Level 2 and 3 of the van Hiele model

\begin{tabular}{|c|c|c|}
\hline & Level 2 & Level 3 \\
\hline Object & Shapes & Properties/theorems \\
\hline Method & Properties & Deductive reasoning \\
\hline
\end{tabular}

Our suggestion is that the study of the classification of quadrilaterals can be used to help promote such changes between object/method in geometrical thinking. To reason successfully about the answer to the question about whether a rhombus is a (special type of) parallelogram, learners need not only to be able to control its image, but also to examine properties (concepts/theorems). In particular, it is likely to be important that learners recognise that a geometrical shape can be seen as 'a collection of properties' (van Hiele, 1986, p. 62), and that some properties can be deduced from other properties (van Hiele calls these 'characteristics'). These activities (examining, listing and ordering properties of geometrical shapes) are likely to be important in the early stages of deductive reasoning. This indicates that the hierarchical classification, 
although difficult, should be included in a geometry curriculum.

\section{Prototype phenomenon and implicit models}

Fischbein et al (1985) found that students' mathematical behaviours in basic arithmetical operations are likely to be influenced by the so-called 'tacit model':

We assume that the models attached to the arithmetical operations are basically behavioral in nature. That is, when trying to discover the intuitive model that a person tacitly associates with a certain operation, one has to consider some practical behavior that would be the enactive, effectively performable counterpart to the operation ... we hypothesized that the enactive prototype of an arithmetical operation may remain rigidly attached to the concept long after the concept has acquired a formal status. (pp. 5-6)

In the context of geometrical thinking, a similar situation has been observed: the 'prototype phenomenon' (Hershkowitz, 1990). The key factor is the prototype example, which Hershkowitz describes as follows:

Each concept has one or more prototype examples that are attained first and therefore exist in the concept image of most subjects. The prototype examples were usually the subset of examples that [had] the "longest" list of attributes - all the critical attributes of the concept and those specific (non-critical) attributes that had strong visual characteristics. (p. 82)

Thus, we speculate that learners are likely to regard, for example, a parallelogram as 'a quadrilateral whose opposite sides are parallel to each other' (definition), and they implicitly add properties such as 'in parallelograms, the adjacent angles are not equal', which are likely to be the result of the prototype of images of parallelograms (see Okazaki and Fujita, 2007). These properties also function as their implicit models and influence their behaviours. As we have seen, learners from our first data set (see Table 2 and Figure 1) are likely to regard a rectangle as 'a quadrilateral which has 2 longer sides and 2 shorter sides'; further, 40 of 76 students from our second data set (see Tables 3 and 5) used limited images of a parallelogram (as in Figure 3) when they tackled the question 'Is it possible to draw a parallelogram whose four vertices are on the circumference of a circle?' We speculate that a reason for this behaviour is that they implicitly add 'rectangles don't have all equal sides', or 'parallelograms don't have right angles' based on their limited images, that is on prototype phenomenon.

Future research could be designed to reveal more precisely what (implicit) images learners have by utilising - for example, by utilising the form of questions presented in Table 7 (see also Nakahara, 1995; Okazaki and Fujita, 2007).

In this exemplar questionnaire, if a learner, for instance, excludes rectangles and squares from the Image question (see the first part of Table 7), then s/he is likely to put ( $\mathrm{X}$ ) for the Property question part (f) (this means 'no' for this question). This would be taken as indicating that s/he is influenced by the prototype image of parallelograms and implicitly adds the property 'parallelograms don't have all equal 
angles'. This would influence her/his mathematical thoughts such as defining, classifying, problem solving in quadrilaterals and so on (see, Okazaki and Fujita, 2007).

In addition to revealing what (implicit) images learners have, it is also important to investigate how we design tasks/teaching sequences that might support learners in overcoming these implicit additional properties of geometrical figures.

Table 7: A possible future research questionnaire

Image question
In the following quadrilaterals (the shapes with the thick black lines), next to each
one, put $(\sqrt{ })$ for those you think are in the parallelogram family, $(\mathrm{X})$ for those you
think do not belong to the parallelogram family, or if you are not sure, put ( ? )
Property question
Read the following sentences carefully, and put ( $\sqrt{ }$ ) for those you think are correct,
(X) for those that are incorrect, and, if you are not sure, put (?)
Questions about Parallelograms
(a) ( ) The lengths of the opposite sides of parallelograms are equal.
(b) ( ) There are no parallelograms which have equal adjacent sides.
(c) ( ) The opposite angles of parallelograms are equal.
(d) ( ) There are parallelograms which have equal adjacent angles.
(e) ( ) There is a parallelogram which has all its sides equal.
(f) ( ) There is a parallelogram which has all equal angles.

\section{Common cognitive path in the understanding of the relationship between quadrilaterals}

From the data analysis presented in this paper, we speculate that a hierarchical order of difficulties might exist among the understanding of the relationship between quadrilaterals. For example, rhombuses might be more likely to be recognised as special types of parallelograms than squares or rectangles, because the former 'look like' parallelograms. As our data indicate, whereas $36 \%$ of learners from our second data set (see Table 5) could answer 'A rhombus is a special type of parallelogram' correctly, just $13 \%$ of those from our first data set knew that 'a square is a rectangle' (see Table 2). Also, learners from our second data set had particular difficulties in solving Q3 (see Tables 3 and 5), where, to be correct, they should have regarded 'a rectangle as a parallelogram'.

Given our conjecture that there might an order of difficulties in the relationships between quadrilaterals, it might not be effective, for example, to teach the 
relationship rectangles/parallelograms before learners fully grasp the relationship between rhombuses/parallelograms. Thus, another important issue for future research is to find 'common cognitive paths' (Vinner and Hershkowitz, 1980) in the cognitive development of quadrilaterals of learners something which Vinner and Hershkowitz describe as follows:

Let us denote by A, B, C, D, four different aspects (components) that partially form a certain concept (or concept image). Assume that a certain learner acquired these aspects in the order they were written above. Then $\mathrm{A} \rightarrow \mathrm{B} \rightarrow \mathrm{C} \rightarrow \mathrm{D}$ is a cognitive path of the above concept for the above learner. Now assume there is a group of people such that $\mathrm{A} \rightarrow \mathrm{B} \rightarrow \mathrm{C} \rightarrow \mathrm{D}$ is a cognitive path for this group. Then we will say that it is a common cognitive path for everybody in the group. (Vinner and Hershkowitz, 1980, p.182)

Several researchers have already found some common cognitive paths in geometrical shapes. For example, Vinner and Hershkowitz (1980) investigated common cognitive paths for obtuse and straight angles, right-angled triangles, and the altitude in a triangle. Nakahara (1995) investigated quadrilaterals and found a common cognitive path for basic quadrilaterals, parallelogram $\rightarrow$ rhombus $\rightarrow$ trapezium, among Japanese primary school children. In future research, we suggest it would be worthwhile investigating what common cognitive paths in each quadrilateral might exist for other groups of learners (such as trainee elementary school teachers) - for example, 'square and parallelogram', 'rhombus and rectangle' and so on - as this is likely to be useful when determining teaching materials and their sequences in the teaching and learning of geometry (see, also, Okazaki and Fujita, 2007).

\section{CONCLUDING COMMENTS AND THE THEORETICAL FRAMING OF FURTHER RESEARCH}

This paper presents an attempt to clarify what knowledge learners have of a particular mathematical topic. As our analysis demonstrates, learners studied for this project show limitations in their knowledge of basic geometrical figures. In the previous section we attempted to synthesise existing research considerations and, as a conclusion, we suggest that future research in the understanding of the relationship between quadrilaterals might be framed as follows:

- the study of the hierarchical classification of quadrilaterals might be regarded as an area of study which would help to promote the development of geometrical thinking from level 2 to 3 of the van Hiele model;

- given that the understanding of the hierarchical relationship is difficult for many learners, with, in particular, their thinking likely to be influenced by prototype images of quadrilaterals (something which might result in a 'longest' list of attributes), it is necessary to reveal what implicit properties learners have in terms of the basic quadrilaterals;

- in order to design an effective geometry curriculum, identifying common cognitive paths of understanding of the basic quadrilaterals among learners may be helpful. 
Studying the usefulness of the hierarchical classification of quadrilaterals as a means of bridging the 'gap' between van Hiele levels 2 and 3, revealing what implicit properties learners have in terms of the basic quadrilaterals, and identifying the common cognitive paths of understanding of the basic quadrilaterals among learners constitute our proposal for a theoretical framing for further research in this area.

\section{ACKNOWLEDGEMENT}

We would like to thank Prof. Kunimune, Shizuoka University, Japan, who kindly allowed us to use his questionnaire (see Kunimune 2000) and provided us with further information about his study undertaken in Japan. The section of our paper entitled 'Towards a theoretical framework' is influenced by Prof. Okazaki's studies (written in Japanese) and we would like to thank Prof. Okazaki for providing us with valuable information on his studies (see Okazaki, 1995, 1999; Okazaki and Iwasaki, 2003; Okazaki and Fujita, 2007, the latter being in English) We would also like to thank Stuart Rowlands and Nick Pratt (both of the University of Plymouth) who kindly read an early draft of this paper and gave us valuable advice; plus we would like to express our thanks to the formal reviewers of this paper for their insightful advice and the RME editors for their attention to detail. Finally, we would like to thank Willie Magill, Gerry Doyle, and Pat Brown (all of the University of Glasgow) for their support and we acknowledge the University of Glasgow who supported this research project during the period 2004-6.

\section{REFERENCES}

Battista, M. T. (2007) The development of geometric and spatial thinking, in: F. Lester (Ed) Second Handbook of Research on Mathematics Teaching and Learning (Charlotte, NC: NCTM/Information Age Publishing).

Crowley, M. L. (1987) The van Hiele model of the development of geometric thought, in: M. M. Lindquist (Ed) Learning and Teaching Geometry, K-12 (Reston, VA: NCTM).

Currie, P. \& Pegg, J. (1998) Investigating students understanding of the relationships among quadrilaterals, in: C. Kanes, M. Goos and E. Warren (Eds) Teaching Mathematics in New Times, Proceedings of the Annual Conference of the Mathematics Education Research Group of Australia, 1, 177-184.

de Villiers, M. (1994) The role and function of a hierarchical classification of quadrilaterals, For the Learning of Mathematics, 14(1), 11-18.

de Villiers, M. (1998) To teach definitions in geometry or teach to define? Proceedings of the $22^{\text {nd }}$ Conference of the International Group for the Psychology of Mathematics Education, 2, 248-255.

Erez, M. \& Yerushalmy, M. (2006) "If you can turn a rectangle into a square, you can turn a square into a rectangle": young students' experience the dragging tool, International Journal of Computers for Mathematical Learning, 11(3), 271-299. 
Fischbein, E. (1993) The theory of figural concepts, Educational Studies in Mathematics, 24(2), 139-162.

Fischbein, E., Deri, M., Nello, M. S. \& Marino, M. S. (1985) The role of implicit models in solving verbal problems in multiplication and division, Journal for Research in Mathematics Education, 16(1), 3-17.

Fujita, T. \& Jones, K. (2003a) The place of experimental tasks in geometry teaching: learning from the textbooks design of the early 20th Century, Research in Mathematics Education, 5, 47-62.

Fujita, T. \& Jones, K. (2003b) Interpretations of National Curricula: the case of geometry in Japan and the UK, paper presented at the 2003 BERA Conference, Heriot-Watt University, September 2003.

Fujita, T. \& Jones, K. (2006) Primary trainee teachers' understanding of basic geometrical figures in Scotland, Proceedings of the 30th Conference of the International Group for the Psychology of Mathematics Education, 3, 14-21.

Hershkowitz, R. (1990) Psychological aspects of Learning Geometry, in: P. Nesher and J. Kilpatrick (Eds) Mathematics and Cognition (Cambridge, Cambridge University Press).

Jones, K., Mooney, C. \& Harries, T. (2002) Trainee primary teachers' knowledge of geometry for teaching. Proceedings of the British Society for Research into Learning Mathematics, 22(2), 95-100.

Kawasaki, M. (1992) A study on the influences of visual imagery in the teaching of geometry, in: Iwago, K. (Ed.). The New Perspective of Mathematics Education (Japan, Seibunsya). [in Japanese]

Koseki, K. (Ed) (1987) The Teaching of Geometrical Proof (Tokyo, Meiji Tosho Publishers) [in Japanese].

Kunimune, S. (2000) A change in understanding with demonstration in geometry, Japan Society of Mathematical Education, Journal of Japan Society of Mathematical Education. Mathematical education, 82(3). 2-12. [in Japanese]

Markman, E. M. (1991) Categorization and Naming in Children: problems of induction (Cambridge, MA: MIT Press). Revised edition.

Monaghan, F. (2000) What difference does it make? Children views of the difference between some quadrilaterals, Educational Studies in Mathematics, 42(2), 179-196.

Nakahara, T. (1995) Children's construction process of the concepts of basic quadrilaterals in Japan, Proceedings of the $19^{\text {th }}$ Conference of the International Group for the Psychology of Mathematics Education, 3, 27-34.

Okazaki, M. (1995) A study of the growth of mathematical understanding based on the equilibration theory: an analysis of interviews on understanding inclusion 
relations between geometrical figures, Japan Academic Society of Mathematics Education, Research in Mathematics Education, 1, 45-54. [in Japanese]

Okazaki, M. (1999) Basic study on activities of defining geometrical figures referring to an investigation on the understanding of inclusion relations between geometrical figures, Japan Academic Society of Mathematics Education, Research in Mathematics Education, 5, 101-110. [in Japanese]

Okazaki, M. \& Fujita, T. (2007) Prototype phenomena and common cognitive paths in the understanding of the inclusion relations between quadrilaterals in Japan and Scotland, Proceedings of the 31st Conference of the International Group for the Psychology of Mathematics Education, 4, 41-8. South Korea, July 2007.

Okazaki, M. \& Iwasaki, H. (2003) Geometric construction as an educational material mediating between elementary and secondary school mathematics: theory and practice to promote transformation from empirical to logical recognition, Japan Society of Mathematical Education, Report on Mathematical Education, 80, 3-27. [in Japanese]

Pickreign, J. (2007) Rectangle and rhombi: how well do pre-service teachers know them? Issues in the Undergraduate Mathematics Preparation of School Teachers, 1, Content Knowledge. Available online at: http://www.k-12prep.math.ttu.edu (published February 2007; accessed 18 June 2007).

Poincaré, H. (1914) Mathematical definitions and education, in: Poincaré, H, Science and Method (trans. F. Maitland) (London, Thomas Nelson). [originally published in France in 1908]

Royal Society (2001) Teaching and Learning Geometry 11-19 (London, Royal Society/Joint Mathematical Council).

Senk, S. L. (1989) van Hiele levels and achievement in writing geometry proofs, Journal for Research in Mathematics Education, 20, 309-321.

Tall, D. O. \& Vinner, S. (1981) Concept image and concept definition in mathematics, with special reference to limits and continuity, Educational Studies in Mathematics, 12(2), 151-169.

Usiskin, Z. \& Dougherty, B. J. (2007) The Classification of Quadrilaterals: a study in definition (Charlotte, NC: Information Age Publishing).

van Hiele, P. M. (1999) Developing geometric thinking through activities that begin with play, Teaching Children Mathematics, 5(6), 310-6.

van Hiele, P. M. (1986) Structure and Insight: a theory of mathematics education (Orlando: Academic Press).

Vinner, S. (1991) The role of definitions in the teaching and learning of mathematics, in: D. O. Tall (Ed) Advanced Mathematical Thinking (Dordrecht, Kluwer Academic Publishers). 
Vinner, S. \& Hershkowitz, R. (1980) Concept images and some common cognitive paths in the development of some simple geometric concepts, Proceedings of the 4th Conference of the International Group for the Psychology of Mathematics Education, 177-184.

Zaslavsky, O. \& Shir, K. (2005) Students' conceptions of a mathematical definition, Journal for Research in Mathematics Education, 36(4), 317-346. 